\title{
Quelques techniques pour l'étude et la mesure du débit solide
}

\section{Methods for the study and measurements of solid discharges}

\author{
PAR G. BRAUDEAU

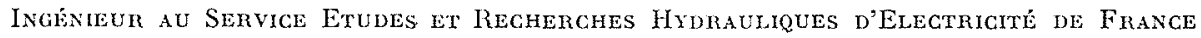

English synopsis p. 217

\section{I. - Introduction}

Le transport des matériaux solides par les eaux courantes entraine, pour des installations hydrauliques très diverses, des difficultés d'exploitation si graves qu'elles font, parfois, renoncer à leur aménagement. Elles se traduisent souvent par des charges financières si lourdes qu'elles justifient des études coûteuses.

En de telles études, le modèle réduit est un moyen d'investigation précieux. Or, le phénomène dépend d'un si grand nombre de paramètres que sa complexité, comme sa variabilité, sont immenses. Aussi sa construction et son exploitation doivent-elles être souvent guidées par des mesures in situ suffisamment complètes pour fixer les conditions aux limites et vérifier que, dans les conditions particulières de réglage, la schématisation des phénomènes est acceptable.

Destinées à étayer l'exploitation d'une maquette d'estuaire à marée ou de chenal navigable, à estimer la viabilité d'un aménagement hydro-électrique, à prévoir ses conséquences agricoles, à fixer les caractéristiques d'une prise d'eau, ou à préjuger de l'efficacité d'un reboisement, les mesures de débit solide sont limitées par des difficultés bien plus financières que spécifiquement techniques.

\section{II. - Mesures de transport solide en suspension}

Le caractère principal du phénomène est sa très grande irrégularité et l'impossibilité, dans la plupart des cas, de relier le débit à la concentration moyenne.

Si l'on ne veut s'exposer à de très importantes erreurs dues à des interpolations injustifiées, il est donc nécessaire d'effectuer des mesures extrêmement fréquentes.

Voici quelques exemples :

On possède des relevés journaliers de débit et de concentration à Mirabeau, sur la Durance, pour une vingtaine d'années (1868-1888). Si l'on admet que ces deux grandeurs varient linéairement entre les instants de mesure, on peut calculer que la crue du 8 novembre 1871 apporta 4.500 .000 tonnes de boue. Si les mesures avaient été décalées de 12 heures, on en aurait trouvé 3.000 .000 , soit en moins 1.500 .000 tonnes. Cette différence représente environ $15 \%$ du transport solide de toute l'année 1871.

Nous trouvons dans un article de l'Américain Collins (Memorandum of the U.S. Geological 
Survey - Water Research Branch, Mai 1939) les courbes indiquant les variations du débit et de la concentration lors d'une crue du Coon Creek (Visconsin) le 10 septembre 1938. Le débit passe de $2,7 \mathrm{~m}^{3} / \mathrm{s}$ à $0 \mathrm{~h}$ à $66 \mathrm{~m}^{3} / \mathrm{s}$ à $4 \mathrm{~h}$, redescend à $6,5 \mathrm{~m}^{3} / \mathrm{s}$ à midi et à $2,7 \mathrm{~m}^{3} / \mathrm{s}$ à minuit. La concentration de $3 \mathrm{~g} / \mathrm{l}$ à $0 \mathrm{~h}$ passe à $30 \mathrm{~g} / 1$ à $1 \mathrm{~b}$ et redescend régulièrement à $0,5 \mathrm{~g} / 1$ à minuit.

L'auteur montre que si l'on avait eflectué un seul prélèvement et multiplic la concentration par le débit au même instanl, on aurait fait, sur le débit solide total annuel, une erreur variant entre -25 et $+120 \%$ selon l'heure de la mesure. En tenant compte du débit moyen de la journée, l'erreur aurait été comprise entro - 25 ei $+60 \%$.

Si les mesures avaient été faites à intervalles de $6 \mathrm{~h}$, on aurait eu, sur le débit solide de la journée, des erreurs variant entre $+60^{\circ}$ et - $80 \%$, selon l'heure de la première mesure.

Nous savons, d'autre part, que dans une section déterminée, les conditions hydrauliques peuvent être telles que la concentration soit loin d'être homogène.

Si le calcul peut fournir, dans certains cas, quelques indications sur la répartition de ces concentrations, il est souvent risqué de se limiter, sans étude préalable approfondie, à un point de mesure où la concentration serait sensée représenter une moyenne.

Cette grande variabilité des concentrations dans le temps et dans l'espace est considérablement accentuée dans le cas d'un estuaire à marée. La nécessité de mesures aussi continues et nombreuses que possibles devient alors inćluctable, si l'on désire une image, même grossière, des phénomènes.

Dans tous les cas, un grand nombre de points de mesure est nécessaire. Si ces mesures doivent être faites par prélèvement d'échantillons et si ces échantillons doivent être analysés de façon précise, le prix de revient du dépouillement devient largement supérieur à celui des campagnes de mesures elles-mêmes et peut être tout à fait prohibitif. On doit alors se poser la question suivante :

Est-il préférable de réduire le nombre et la cadence des mesures, ou de procéder à des analyses plus sommaires?

\section{b) Mesures en estuare. - Analyses volumé- TRIQUES.}

Deux campagnes de mesures, menées par la Div. Ess. Ext. du S.E.R.H. nous ont permis de nous faire une opinion à ce sujet; l'une en Loire-Maritime, en juillet 1949, l'autre en Gi- ronde, en avril 1950, dont le dépouillement est en voie d'achevement. 'Toutes deux avaient pour but de préciser les conditions de transport des vases le long des chenaux desservant respectivement les ports de Nantes et de Bordeaux, afin d'étayer les études sur modèles, effectuées par le laboratoire de Chatou.

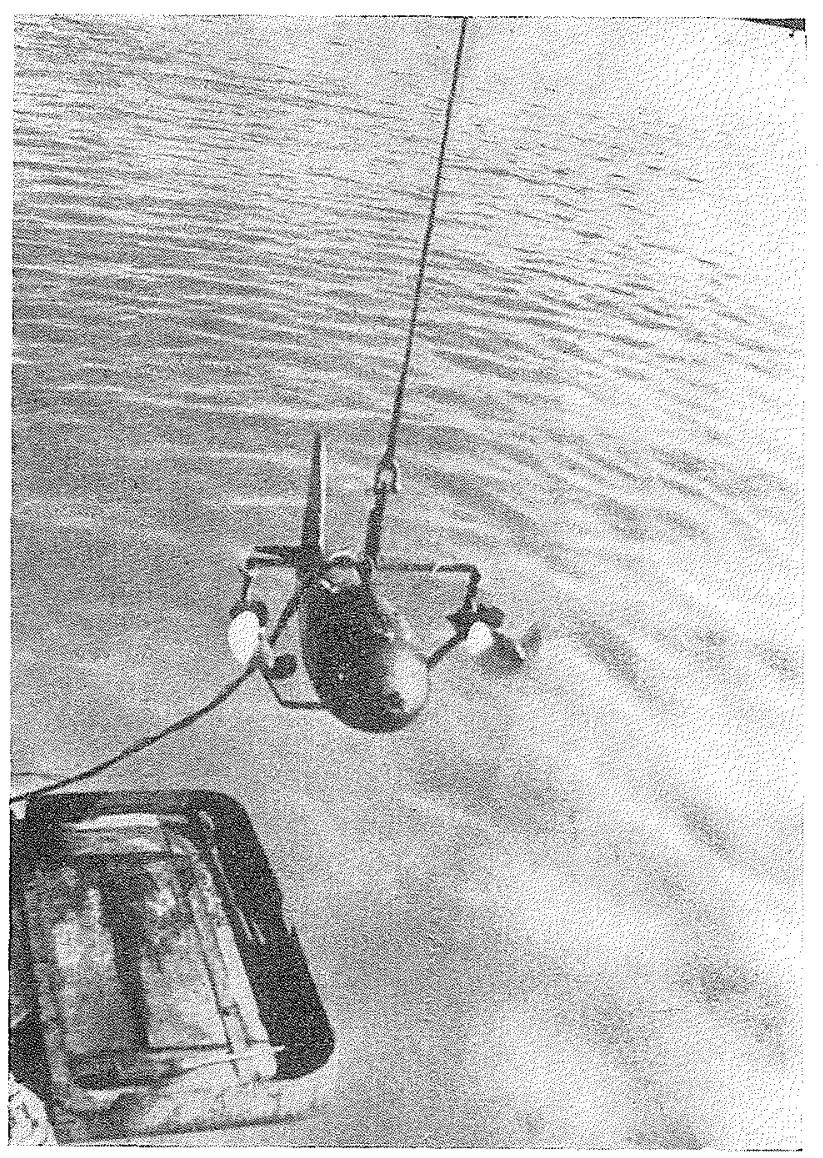

Fig. 1. - SAMPler P. 46 et Mothinets villisés EN LOIRE-MartTMU ET EN GIRONDE.

La première de ces campagnes nous a permis de mettre au point une technique d'analyse, sans laquelle la seconde eut été financièrement impossible.

En Loire-Maritime, nous avons choisi treizo verticales de mesure de la façon suivante : neuf étaient échelonnées le long de la passe, sur environ $45 \mathrm{~km}$. Leur espacement était compris entre 4 et $8 \mathrm{~km}$. Deux ont été placées pour compléter le profil en travers de Montoir, deux autres pour compléter celui de la Maréchale.

Sur chacune de ces verticales ont été effectués pendant une marée complète (13 heures pour obtenir un léger recouvrement) des prélèvements à hauteurs différentes, de 3 à 7 selon le tirant d'eau. Les séries de prélèvements étaient effeclućes, en moyenne, toutes les 20 minutes. A 
chaque point de prélèvement, la vitesse était mesurée.

Trois équipes nous ont permis d'examiner 3 verticales chaque jour, et de mener l'ensemble de la campagne en cinq jours, choisis pour de très faibles variations des coefficients de marée.

Plus de 2.000 échantillons prélevés devaient être analysés. Cette analyse devait comporter :

- dosage des matières solides,

- dosage des sels dissous,

- analyse granulométrique des échantillons prélevés aux points qui, après dépouillement, se seraient avérés particuliers.

Les échantillons, contenus dans des bouteilles, étaient laissés au repos 24 heures. L'eau en était alors aspirée, son volume étaït noté et sa salinité mesurée par une cellule à résistivité.

Cette eau était remplacée par de l'eau douce qui, après 24 heures de décantation, était ellemême aspirée et remplacée. L'échantillon, convenablement agité, était alors versé dans un tube de verre, à base rétrécie, gradué en volume, conformément au croquis ci-joint (fig. 2). Les volumes déposés dans les tubes étaient lus à intervalles de temps déterminés, échelonnés jusqu'à 23 heures après la mise en décantation.

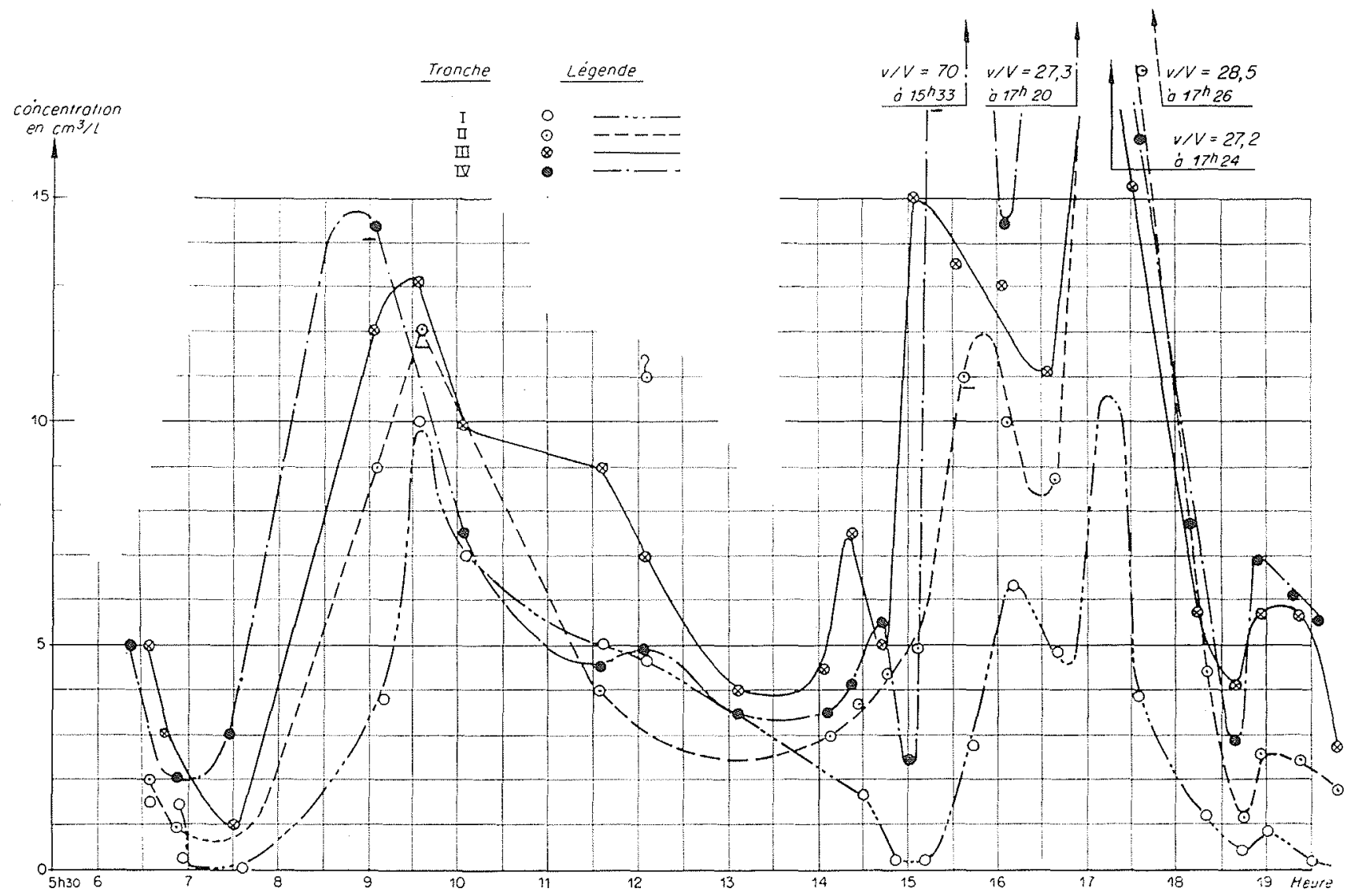

Fig. 3. - Exemple de diagramme des concentrations omtenues en lome-Maritime.
Ces volumes commencaient par crôttre, atteignaient un maximum après 5 heures environ,

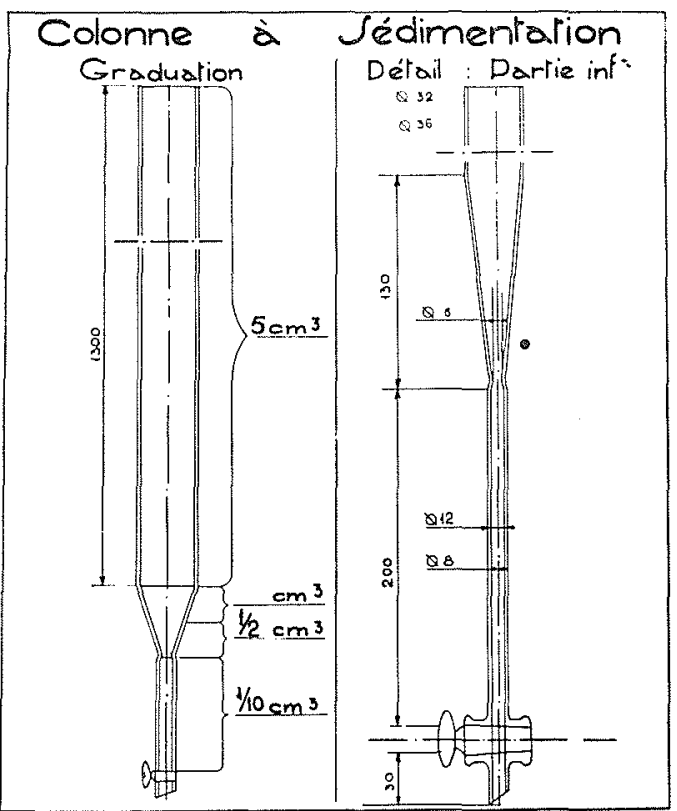

Frg. 2

et décroissaient par suite de l'influence prépondérante du tassement. 
Les dépôts étaient recueillis. Un échantillon sur cinq, après dessiccation, était pesé. Le poids était divisé successivement par les différents volumes lus pour le même échantillon après les cinq premières heures. On obtenait ainsi une série de densités apparentes, correspondant chacune à un certain temps de décantation et de tassement. On a pu constater que les densités apparentes les plus voisines, pour tous les échantillons pesés, étaient calculées avec les volumes lus après 5 heures de décantation, c'est-à-dire avec les volumes maxima. Pour ces densités, l'écart probable était de l'ordre de $13 \%$ autour d'une valeur moyenne de 0,15 .

Or, l'examen des diagrammes tracés pour la Loire-Maritime, indiquant en fonction du temps et pour chaque verticale, la variation des concentrations dans les différentes tranches de l'écoulement montre que, manifestement, les variations désordonnées des concentrations, en un point et

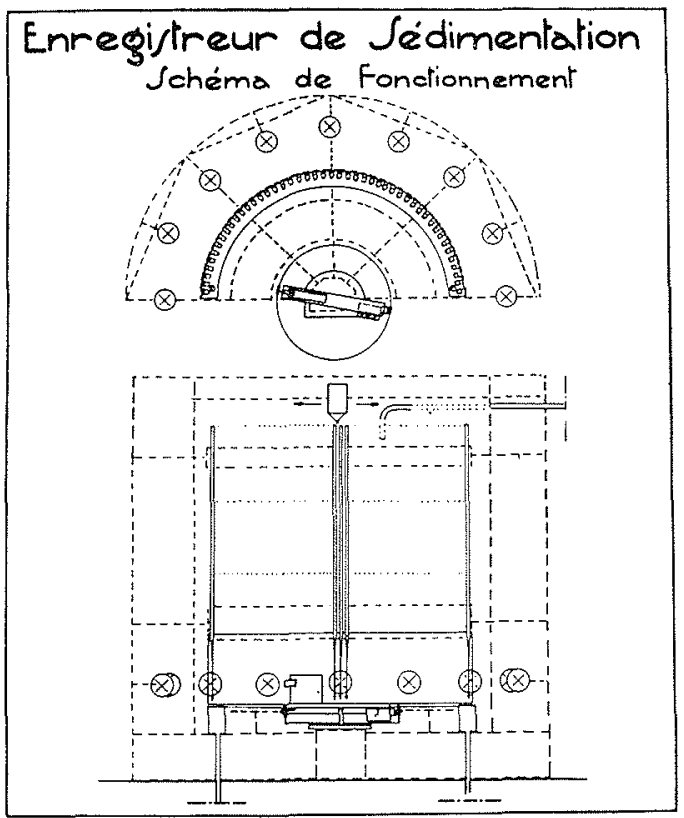

FIG. 4

à un moment donnés, autour d'une valeur probable, sont très largement supérieures' à la marge d'erreur admise sur les dosages. Il montre, par contre, que, étant donné les variations énormes, dans le temps et dans l'espace, de ces concentrations, réduire le nombre et la cadence des mesures nous aurait masqué la vue d'ensemble assez complète du phénomine que nous avons pu obtenir.

Les mesures effectuées en Gironde en avril 1950 ont largement bénéficié de l'expérience acquise sur la Loire, tant pour la répartition des points de mesure (tranches de mêmes hau- teurs relatives rapportées au tirant d'eau) que pour l'analyse des 4.000 échantillons prélevés (24 verticales) et la présentation des résultats.

L'analyse des échantillons prélevés en Gironde s'acheve dans des conditions identiques. On ne pèse qu'un échantillon sur dix. On a pu déterminer que, dans ce cas, les volumes lus après 23 heures de décantation fournissaient les resultats les plus comparables. Les densités ainsi calculées présentent, autour de leur moyenne, un écart probable de l'ordre de $10 \%$;

Cette méthode permet d'analyser au moins 50 échantillons chaque jour. Contrairement aux filtrations et pesées couramment pratiquées, elle n'exige aucun matériel coûteux et, surtout, n'occupe que peu de temps un personnel non spécialisé.

Pour la rendre plus industrielle encore, nous avons réalisé un montage dont le principe est fort simple : 50 tubes d'analyse sont disposés sur un demi-cylindre, au centre duquel pivote, lentement, une caméra. Les tubes, éclairés en contre-jour à travers un écran de rhodoïd, par des lampes s'allumant deux par deux dans le champ de l'objectif, sont photographiés par groupe de 4, avec recouvrement d'un tube sur deux photographies successives. La mise en route et l'arrêt du dispositif peuvent être commandés par une horloge mère et le film indiquera les volumes déposés aux intervalles de temps choisis. La présence d'un opérateur n'est exigée qu'une heure environ chaque jour, pour le remplissage et le lavage des tubes. Le dépouillement des films pourra se faire globalement, au bureau.

La variation du volume déposé dans chaque tube pourra, de plus, fournir parfois des indications qualitatives intéressantes sur la granulométrie des matériaux.

c) Mesure eN RIVIERE, PAR PRÉlÈveneNT CONTINU.

Nous avons vu combien peuvent être grandes les erreur's dues à une trop faible cadence des mesures. Les variations brutales de concentralions se produisent surtout au moment des crues qui font, en général, passer, en quelques jours, dans une section, la quasi-totalité du débit solide annuel d'une rivière. (Pour la Durance, à Mirabeau, $90 \%$ du débit solide annuel passe en une quinzaine de jours). A ces moments, des prélèvements devraient être effectués, au moins toutes les $1 / 2$ heures. Sur une rivière où les crues sont brutales et peuvent être échelonnées 
sur plusieurs mois, l'immobilisation totale du per'sonnel nécessaire, en état d'alerte, est pratiquement impossible.

Pour s'affranchir de cette sujétion, nous avons réalisé un appareil dont la mise au point et le réglage se poursuivent à Mirabeau, sur la Durance. Son but est d'effectuer un prélivemenl continu et d'intégrer automatiquement le débit solide passant chaque jour en suspension dans la section de mesure. Un certain nombre de causes d'erreurs ont été acceptées, au profit de la continuité des mesures et de l'automaticité de la marche.

Le principe en est le suivant:

A chaque instant, une quantité d'eau chargèe, prélevée en rivière, proportionnelle au débit a

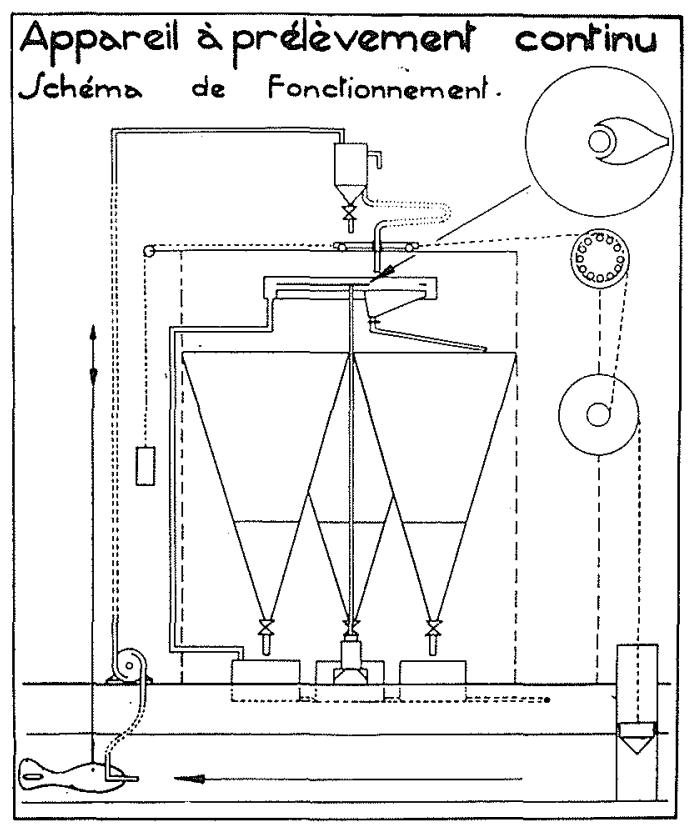

FıG. 5

cet instant, est envoyée dans une cuve conique dont la partie inférieure est transparente. Le volume de la cuve est suffisant pour emmagasiner l'eau prélevée pendant 24 heures, sur un débit de $2.000 \mathrm{~m}^{3}$, correspondant à la crue annuelle.

Il existe trois cuves identiques, ce qui permet à chacune de rester deux jours en décantation. Après décantation, le volume déposé dans unc cuve est une fraction constante et connue du volume de matériaux passés dans la section pendant les 24 heures correspondantes.

La proportionnalité du débit prélevé au débit en rivière est réalisée de la façon suivante :

Un tube de prise, lié à un saumon, est immergé en un point déterminé de l'écoulement. Le pro- blème de la détermination de ce point sera examiné plus toin. Ce tube est relié à l'aspiration d'une pompe volumétrique qui refoule l'eau prélevée dans un bac à niveau constant.

Un tuyau a faible section, souple et long, préleve, dans ce bac, un débit constant qu'il envoie vers un ajutage lić à un chariot. Ce chariot peut décrire, sur deux rails, une course rectiligne de $50 \mathrm{~cm}$. Sous les rails se trouve un large entonnoir, d'où une goulotte peut être dirigée à volonté vers l'une des trois cuves.

Entre les rails et l'entonnoir tourne un disque horizontal percé d'une Iumière, constituant le distributeur de débit.

La position du chariot sur ses rails étant commandée, par l'intermédiaire de poulies réductrices et d'une came à développante, par un flotteur de limnigraphe, la lumière a une forme telle que le temps pendant lequel elle se trouve en regard de l'ajutage, où donc l'eau prélevéc est envoyée vers la cuve, soit proportionnel au débit en rivière.

Le disque est entrainé par un moteur électrique muni d'un réducteur. Il est à noter que les variations de sa vitesse de rotation restent sans influence sur la proportionnalité des débits.

Il est nécessaire, pour déterminer la forme de la lumière et de la came, de connaitre la courbe hauteur-débit de la rivière pour la section choisie. Dans le cas particulier de la Durance, à Mirabeau, nous ne possédions d'une telle courbe aue l'allure très approximative. Un tracé théorique fut choisi et la forme de la lumière calculée pour correspondre à une came circulaire. Cette came, à développante, est constituée par 12 pions pouvant être déplacés sur 12 ravons d'un cercle. Elle pourra ainsi être ajustée, à la demande, selon les corrections apportées à la courbe Hauteurs-Débits.

On peut dresser, des erreurs volontairement acceptées, la liste suivante :

a) Erreur due à la prise unique;

b) Erreur due à une vitesse de prise constante. différente de la vitesse de l'écoulement;

c) Erreur due à la lecture du volume;

d) Erreur due à l'imprécision de la courbe Hauteur-Débit;

e) Erreur due aux frottements mécaniques et au positionnement du chariot.

a) Pour diminuer l'erreur due à la prise unique, il est nécessaire de se placer dans une section aussi turbulente que possible, afin d'éviter de trop fortes variations de concentration d'un point à un autre. 
D'autre part, des campagnes de prélèvements complètes sont à prévoir, soit pour vérifier l'homogénéité de concentration, soit pour déterminer le point où la concentration pourra représenter la concentration moyenne avec la meilleure approximation.

b) La concentration d'un prélèvement est erronée si celui-ci a été effectué à une vitesse différente de la vitesse d'écoulement à l'amont. Toutefois, des études très complètes menćes, à ce sujet, au Laboratoire d'lowa, ont montré qque, pour des matériaux fins (inférieurs à $0,1 \mathrm{~mm}$ ) et des rapports de vitesse n'excédant pas 2 , l'erreur était insignifiante.

La section du tube de prise et le débit de pompage peuvent être facilement calculés pour que le rapport des vitesses n'excède jamais cette valeur.

c) Nous avons déjà examiné les erreurs dues à la lecture du volume. Encore faut-il noter que nous avons pris pour référence les poids de matière sèche et que l'on est en droit de se demander laquelle des deux quantités, poids ou volumes, exacts ou approchés, est la plus significative.

d) L'erreur due à l'imprécision de la courbe $\mathrm{Q}(\mathrm{H})$ subsisterait dans le calcul des débits solides instantanés par prélèvements discontinus.

e) L'appareil a été largement dimensionné pour rèduire l'influence des erreurs de transmission mécanique. Une erreur de $1 \mathrm{~mm}$ sur la position du chariot correspond à une variation de débit de $7 \mathrm{~m}^{3} / \mathrm{s}$. Or, le débit moyen, en Durance, est de $200 \mathrm{~m}^{3} / \mathrm{s}$ environ, et les débits solides importants commencent, en général, aux environs de 600 ou $700 \mathrm{~m}^{3} / \mathrm{s}$. Cette erreur est donc relativement faible.

Nous pensons que ces défauts sont largement compensés par la continuité des mesures, qui supprime la plus grosse cause d'erreurs en ce domaine, et par les très faibles frais d'exploitation: une visite journalière, par une personne sans connaissance spéciale, est suffisante.

\section{d) Mesures en RIVIERE par tUrbidimétrie.}

Si l'on pouvait mesurer la concentration des eaux au sein même de l'écoulement, on aurait, évidemment, loisir de multiplier les points de mesure, étant écartée la grosse sujétion du stockage et de l'analyse des échantillons.

Après bien d'autres, et notamment Rrchard- son, nous nous attachons à résoudre ce problème par l'étude des propriétés optiques des suspensions.

Ricmardson est parvenu, au laboratoire, à relier, de façon biunivoque, la concentration et la transparence, dans des conditions malheureusement limitées: grains uniformes, de diamètres compris entre 6 et 20 microns. Avec quelques approximations, il a pu étendre la méthode à des suspensions de grains non uniformes, mais dont les diamètres restaient compris dans les mêmes limites.

Les bases théoriques sont fragiles. Pour des particules de l'ordre du micron, l'absorption dc la lumière serait proportionnelle à $\Sigma \mathrm{Nd}(\mathrm{N}=$ nombre de particules de diamètre $d$ ). Pour des grains supérieurs à $10 \mu$ elle serait proportionnelle à $\Sigma N d^{2}$.

En réalité, la limite d'application de ces deux lois, elles-mêmes très approximafives, est mal définie. Il est probable que d'autres facteurs que le $\Sigma \mathrm{N} d$ ou $\Sigma \mathrm{N} d^{2}$ interviennent et qu'une mesure de transparence seule ne peut parvenir à déterminer la concentration d'une suspension.

La nature des matériaux, leur granulométrie, sont probablement des facteurs importants.

Pour une rivière déterminée, il arrive souvent qu'en première approximation ces deux facteurs soient liés, des matériaux d'un certain type minéralogique fournissant des tranches granulométriques limitées.

Sans prétendre représenter la granulométric par un paramètre, on est en droit de penser que l'addition d'unc seconde équation faisant intervenir la concentration permettra de mieux déterminer celle-ci.

Or, la transparence étant mesurée par la réponse d'une cellule photo-électrique recevant un faisceau d'une source lumineuse à travers la suspension, il est facile, en plaçant une seconde cellule à $90^{\circ}$, de mesurer la quantité de lumière diffractée. Il n'est pas impossible que l'ẻlimination, entre les deux relations obtenues, d'un paramètre secondaire important, permette de déterminer la concentration avec une précision acceptable.

Si l'on appelle $\mathrm{L}_{1}$ et $\mathrm{L}_{2}$ les quantités de lumière venant d'une même source et respectivement transmise et diffractée à $90^{\circ}$, la vérification de nos hypothèses se fera si, en première approximation, nous pouvons tracer une famille de courbes en $L_{1}$ et $L_{2}$ à un paramètre (concentration $\gamma$ ) ne se recoupant pas.

Pour un sédiment donné, cette famille de courbes représentera le tarage de la méthode. On peut alors penser que des changements dans la composition minéralogique se traduiraient par 
de simples changements d'échelle, linéaires si les courbes sont tracées en coordonnées logarithmiques, puisqu'ils feraient varier les coefficients d'absorption de la matière intervenant généralement sous forme exponentielle dans les equations de la lumière.

Il faut noter que notre but est d'opérer un tarage purement empirique des appareils réalisés, pour éviter de se baser sur des lois incertaines et de tenir compte des perturbations introduites par les appareils eux-mêmes.

Deux appareils sont construits :

Un appareil de laboratoire est destiné plus particulierement à l'étude de principe de la méthode et au tarage. Il se compose d'un élément tubulaire transparent à section carrée de $4 \mathrm{~cm}$ de côté. Devant trois faces successives sont placées une lampe de 50 watts et deux cellules photoélectriques à couche d'arrêt, pouvant attaquer directement un microampèremètre ou bien être montées, avec un galvanomètre, en opposition avec une pile étalon sur un circuit potentiométrique.

Cet ensemble est placé sur un circuit de tuyauterie comprenant en série une pompe et une cheminée pour l'introduction des matériaux.

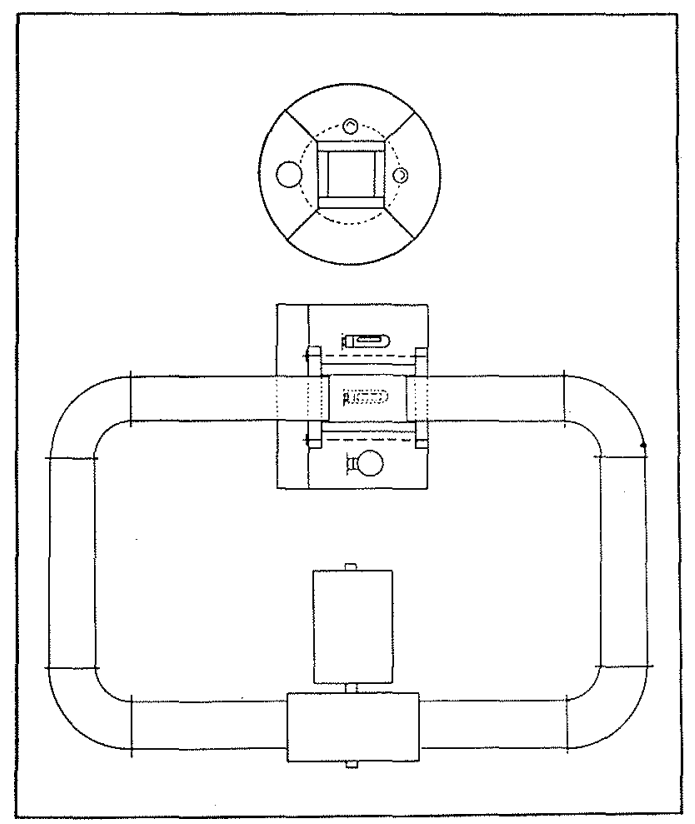

FIG. 6

Nous projetons de commencer l'étude en faisant varier la concentration de suspensions de grains uniformes. Différents diamètres de grains seront utilisés successivement.

Nous' réaliserons, ensuite, des mélanges déter- minés, dont nous ferons, de la même manière, varier la concentration.

La composition des mélanges sera progressivement compliquée, jusqu'à atteindre celle des sédiments naturels.

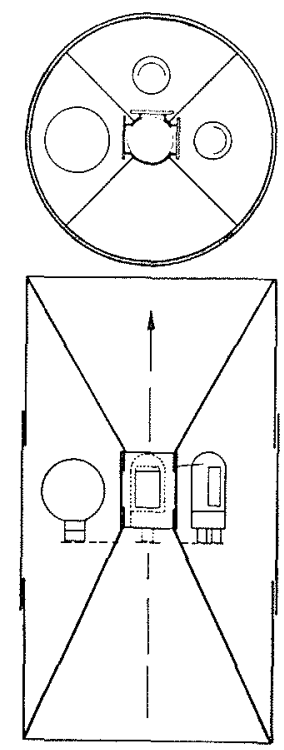

Fig. 7

Un appareil immersible comprend le même élément transparent que celui décrit ci-dessus. Cet élément forme le col d'un venturi, recouvert extérieurement par un capot étanche sous lequel sont abritées lampe et cellules.

Son utilisation en rivière, coordonnée avec des prélèvements et des analyses, permettra de compléter le tarage de la méthode et de fournir, dès maintenant, des indications qualitatives continues' sur les variations de concentration.

Quels que soient les résultats de cette étude, on peut, dès maintenant, songer à utiliser de tels dispositifs, même sous leur forme simple à une seule cellule, pour équiper, en amont des sections de prélèvement, des stations d'alerte permettant aux opérateurs d'effectuer des prélèvements à cadence accélérée dès l'arrivée du flot turbide.

\section{III. - Mesures de charriage}

\section{a) GÉnéraltutes.}

Lorsqu'une rivière coule sur ses propres alluvions, on doit admettre qu'il existe une relation 
entre la quantité de matériaux qu'elle charrie et les caractéristiques hydrauliques de l'écoulement, c'est-à-dire le débit s'il n'y a pas de modification profonde du profil en long.

Il s'agit done d'un phénomène beaucoup plus simple que celui du transport en suspension. Les mesures sont, cependant, bien plus difficiles et imprécises.

En ce domaine, les études théoriques et les études sur modèle ont été développées de façon telle qu'elles sont très en avance sur les techniques de mesure en riviere.

\section{b) Mesures DE DÉbits solides charriés.}

Les rares mesures directes sont faites, jusqu'à présent, à l'aide de nasses à gravier, objet de nombreux travaux dont les plus complets sont, sans doute, ceux du Dr H. A. Ernstern (Etalonnage de la nasse employée sur le Rhin, Schweizerische Bauzeitung $\mathrm{n}^{\circ} 12$, Vol. 110). Dans un lit large et tourmenté, c'est là un moyen de mesure difficile et coûteux, si l'on désire une précision acceptable. Il présente l'avantage de rester toujours perfectible, au prix d'opérations plus longues et plus nombreuses.

Ia mesure continue peut ètre faite en barrant la rivière par une fosse, d'où l'on drague en permanence les matériaux venant de l'amont. Cette méthode, théoriquement satisfaisante, a été, jusqu'à présent, peu emplovée en raison de son prix de revient trop élevé. Nous avons connaissance d'une seule installation de ce genre, réalisée par le Dr Ensstern, à une échelle ne s'éloignant guère de celle du laboratoire (H. A. Ensstern. Bed Load Distribution in Mountain Creek. U.S. Department of Agriculture. Soil Conservation Service, Washington, août 1944). Sur une rivière importante, une véritable installation industrielle de dragage devrait être montée, et l'on aurait l'obligation de rejeter à l'aval la totalité des matériaux dragués, si l'on ne veut, en modifiant le profil en long, créer un régime variable où les mesures ne signifieraient plus grand chose. Toutefois, M. REMÉníras, dans une courte étude consacrée à cette question, note cu'il est possible d'éviter cet inconvénient en placant la fosse au voisinage immédiat d'une section de contrôle, située à l'aval d'une zone de régime uniforme et $y$ maintenant une cote fixe. Les frais supplémentạires d'établissement peuvent alors, dans certains cas, etre compensés par la vente des graviers dragués.

A part auelcues cas bien particuliers, on peut dire cu'actuellement l'estimation du débit solide charrié par les rivières est presque toujours basé sur la mesure de leurs apports globaux dans un réservoir, naturel ou artificiel, au cours d'une saison, d'une année ou de plusieurs années. Des sondages précis permettent de cuber ces apports, l'examen des matériaux et de la configuration des dépôts permettent d'y distinguer la partie charriée de celle transportée en suspension.

Une telle estimation peut être suffisante. Mais il est des cas où l'on désirerait connaître la variation du débit solide charrié avec le débit liquide ou, tout au moins, déterminer le débit à partir duquel la rivière commence à charrier.

\section{c) MESURE DU DÉBIT DE COMMENCEMENT D'ENTRAI-} NEMENT. - DÉTECTEUR HYDROPHONIQUE.

La D.E.E. du S.E.R.H. a repris les études faites, en 1942, à Grenoble, d'un détecteur hydrophonique. Le Laboratoire de Beauvert de la S.H.F. en avait fait l'étude hydraulique, sur mođ̀̀le réduit. Après diverses modifications, il avait assuré à cet appareil une bonne stabilité sur le fond. Nous avons donc repris strictement les mêmes formes et les mêmes' dimensions.

Une plaque triangulaire, en laiton, de $75 \mathrm{~cm}$ de côté, forme la hase de l'appareil. Elle est posée sur le fond. Par trois montants acoustiquement isolés, elle supporte, à environ $15 \mathrm{~cm}$ au-dessus d'elle, un boîtier étanche qui, dans l'appareil étudié à Brauvert, contenait le microphone. Celui-ci était attaqué par un ressort à lame, fixè à la base triangulaire.

Dans l'appareil réalisé à Paris, le microphone est placé directement contre la plaque de laiton, sous une petite coupole étanche et profilée. Il attaque un amplificateur, avec écoute sur casque.

Les résultats qui avaient été obtenus à Grenoble, par les services d'aménagement de l'Isère, avec le premier appareil de ce type, avaient été très encourageants. Nous n'en possédons aucune relation écrite.

Anrès quelques observations, peu probantes, au laboratoire. le détecteur fut immergé dans l'Isère, au Pont de Montrigon, les 24 et 25 mars 1950, à l'occasion de mesures de propagations d'intumescence. nour lesquelles on opérait à Viclaire des lâchers importants' : $20 \mathrm{~m}^{3} / \mathrm{s}$ et $40 \mathrm{~m}^{3} / \mathrm{s}$. La variation de débit qui en résultait levait faire passer celui-ci par la valeur à laquelle le fond est mis en mouvement.

Les résultats furent surprenants. Au cours d'une montée de débit, on pouvait "entendre» le phénomène avec une précision comparable à celle de sensations purement visuelles. Chaque 
bruit pouvait ètre distingué : au début, le bruissement de l'eau, les chocs et le glissement de grains de sable isolés. Ensuite, l'arrivée progressive de cailloux de différentes grosseurs, leur roulement ou leurs chocs répétés sur la plaque. Enfin, le débit décroissant lentement, le glissement de plus en plus fréquent des cailloux et le retour, très progressif, à l'état initial. Il est impossible de décrire de telles observations. Si les conclusions que l'on en peut tirer sont très intuitives, elles n'en ont pas moins une valeur certaine.

D'ailleurs, on peut diminuer sensiblement $l^{\circ}$ facteur personnel en enregistrant les sons entendus. Les appareils modernes d'enregistrement sur fil en fournissent un moyen simple et d'utilisation commode.

Dans les conditions de l'essai, on a pu évaluer le débit de commencement d'entrainement à $18,5 \mathrm{~m}^{3} / \mathrm{s}$ à $\pm 1 \mathrm{~m}^{3} / \mathrm{s}$ près. On doit cependant noter que ce débit peut être différent de colui qui, dans les conditions naturelles, met en mouvement les premiers cailloux. En effet. l'onde de débit était assez brutale. Or, le début de charriage est, en réalité. la rupture d'un équilibre plus ou moins stable du fond. Il est probable qu'une augmentation plus progressive du débit aurait permis à cet équilibre de subsister plus longtemps.

\section{Conclusion}

Pour choisir parmi les nombreuses techniques proposées pour l'étude et la mesure du débit solide, on doit obéir à quelçues idées directrices.

Celles que nous nous sommes fixées sont les suivantes :

Remplacer les mesures isolées à grande précision intrinsèque, mais inutilisables sans erreurs importantes, par des mesures continues et nombreuses, individuellement moins précises, mais anportant, au résultat final, une précision statistique.

Rendre ces mesures financierement praticables par la recherche de l'automaticitć et la mise en cuvre par un personnel réduit et non spécialisé.

\section{DIS CUSSION}

Sur deux questions de M. le Président, M. Bravenau, d'une part, précise que la vitesse de l'eau était dans les essais du détecteur hydrophonicue de l'ordre de $0,80 \mathrm{~m}$ par seconde, et d'autre part, confirme que les résultats de l'écoute ont dépassé ses espérances.

M. Gariel loue la communication pour sa forme et pour son fond; il est, comme l'auteur, agréablement surpris que l'acoustique permette de suivre le phénomène aussi bien que visuellement, et croit qu'il est du plus haut intérêt de faire aboutir les méthodes que celui-ci a exposées si clairement.

M. Gmert signale qu'il a acheté en Allemagne un appareil analogue au turbidimètre à cellule décrit par M. Bravneau, construit par la Maison Ascania, et utilisé par le Service Hydrographique Allemand pour les mesures des matériaux en suspension en mer. D'autre part, il signale que la question du transport des matériaux par charriage a un très gros intérêt pour la Loire-Maritime, dont le curage périodique à Nantes, pour les besoins de la navigation, en donne une idée grossière : un ingénieur de Tours, M. Tournier, avait proposé de « fluxer » ces matériaux de manière à les remettre en suspension, en dirigeant sur le fond de la Loire des jets d'eau très puissants; mais ce procédé soulevait des objections théoriques et n'aurait pas été sans doute très efficace pour ce qui concerne tout au moins les sables.

M. Braudeau ajoute que des appareils à me seule cellule, utilisant la transparence. sont utilisés en France, dans certaines installations d'eau potable, mais ils ne donnent que des indications qualitatives (Bassin de Saint-Christophe à Marseille).

La comparaison qui a été faite entre ce que l'ou entend a l'écoulement des calloux et ce que l'on pourrait voir si la vision était possible, conduit M. ArLeEret a se demander s'il ne serait pas possible de mettre deux micros au lieu d'un et de chercher des effets de relief. Ceci ne donnerait rien pour les petits matériaux, mais pour les blocs permettrait de se rendre compte dans quelle mesure ils passent entre les deux micros ou au contraire à l'extérieur. Une oreille exercée arriverait peut-être à tirer beaucoup plus de renseignements de leux micros que d'un seul, et ces renseignements seraient, à son avis, plus objectifs et plus immédiats que ceux que l'on pourait tirer de l'analyse d'un enregistrement.

M. Braudeay dit que cette suggestion pourrait être réalisée sous réserve d'augmenter les dimensions de l'appareil, ou d'utiliser deux appareils; que de toute laçon, l'enregistrement n'a aucun but de mesure par analyse, mais simplement celui d'éliminer le facteur persomel, afin qu'un auditoire plus nombreux et plus varié, et peut-être moins partial, puisse entendre ces phénomènes et confronter ses impressions.

M. le Président ajoute que le relief sonore serait très intéressant mais que les enregistrements ont de toute facon leur utilite pour la formation des observateurs, comme dans la détection des avions ou des sous-marins.

Sur une question de M. Raynaun, M. Braudeau précise que les points de prélèvements dans la Loire-Maritime ont été placés au milieu de la passe navigable sur des verticales et au milieu de tranches dont la hauteur relalive par rapport au tirant d'eau était respectivement $1 / 10,2 / 10,3 / 10,4 / 10$; les tranches étaient plus minces vers le fond en vue de la détection des moments de mise en suspension ou, au contraire, de dépôt. 
M. Waller suggère d'orienter les cellules photo-électriques suivant un angle aigu plutot que droit, afin de capter le plus possible de lumière diffractée au lieu de la lumière réfléchic, et d'accroître ainsi l'importance du parametre geométrique vis-a-vis du paramètre physique (état de surfaces des particules).

M. Braudeau précise que, dans le cas de suspension de grains fins et nombreux, toute la lumière est diffractée, mais elle l'est plus dans la direction du faisceau lumineux; dans ees conditions, deux cellules placées suivant un angle très réduit donneraient des indications moins distinctes, done moins utilisables que si elles sont placées à $90^{\circ}$.

M. Buesel se demande pourquoi on a prévu le passage de la veine par un venturi qui peut créer une accéléra- tion du liquide et un changement de la concentration spatiale des particules en suspension.

M. Bratofar indique que l'appareil a été placé au col du venturi pour lui donner l'encombrement minimum; dans ces conditions l'accélération longitudinale subie par les filets liquides a faible courbure, à son avis, ne change pas pratiquement la concentration.

M. Reménínas remarque que l'effet signalé par M. Breser existe bien mais est négligeable dans le cas ctudié en raison du faible diamètre des grains.

M. Sauvage de SainT-Mare précise que la concentration spatiale est certainement changée, mais que celle des transports ne l'est pas.

M. le Président remercie M. Braudeau pour sa très intéressante communication.

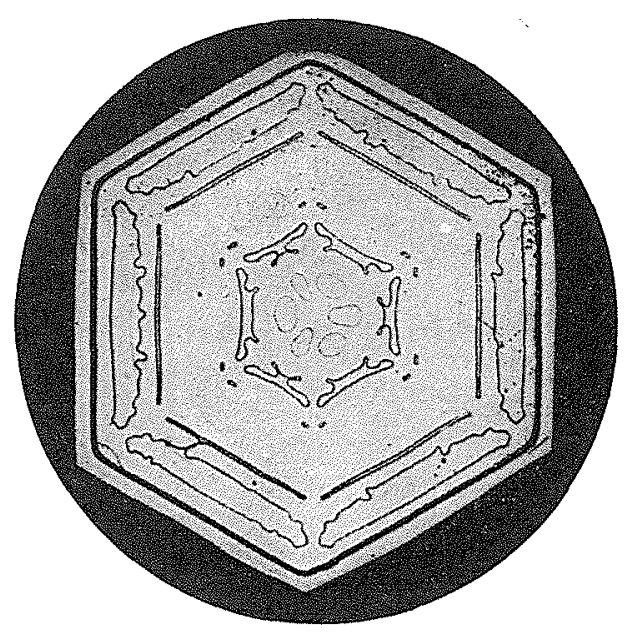

